\title{
What Is the Impact of Ambulatory Vital Sign Monitoring on Deterioration Detection and Related Clinical Outcomes in Hospitalised Patients: a Systematic Review and Meta-analysis.
}

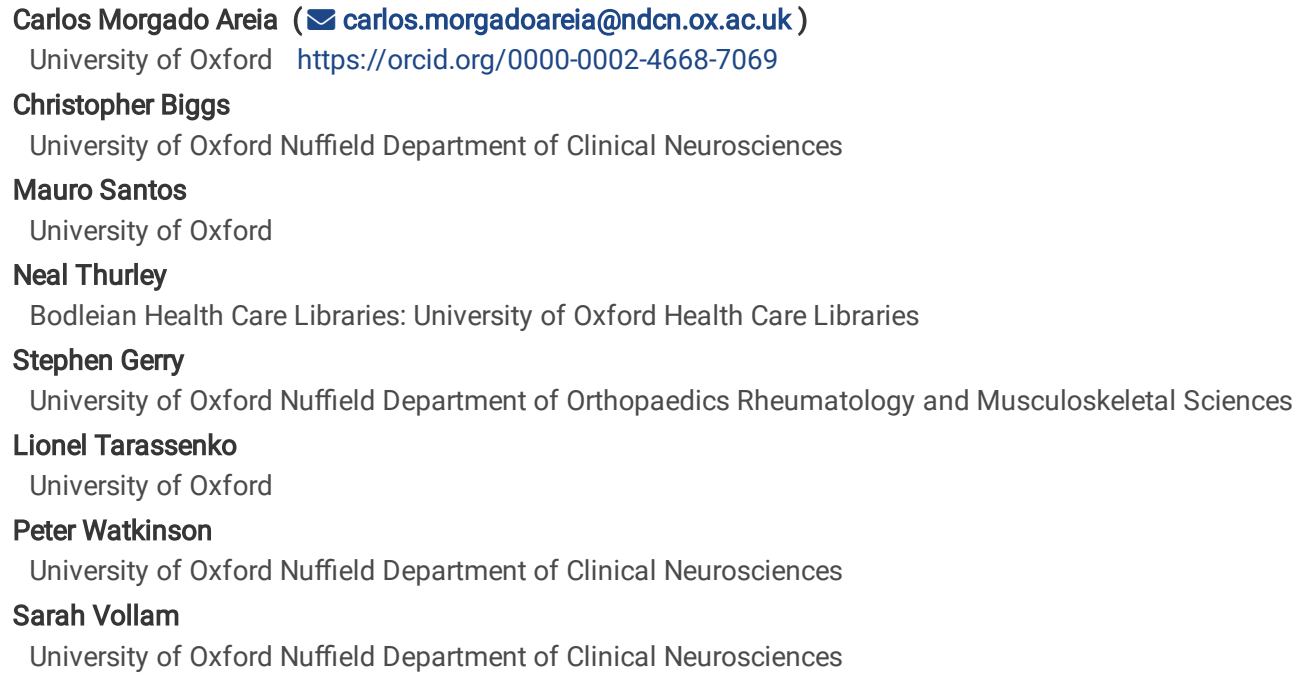




\section{Abstract}

Background: Timely recognition of the deteriorating inpatient remains challenging. Ambulatory monitoring systems (AMS) may augment current monitoring practices. However, there are many challenges to implementation in the hospital environment, and evidence describing the clinical impact of AMS on deterioration detection and patient outcome remains unclear.

Objective: To assess the impact of vital signs monitoring on detection of deterioration and related clinical outcomes in hospitalised patients using ambulatory monitoring systems, in comparison with standard care.

Methods: A systematic search was conducted in August 2020 using MEDLINE, Embase, CINAHL, Cochrane Database of Systematic Reviews, CENTRAL and Health Technology Assessment databases, as well as grey literature. Studies comparing the use of AMS against standard care for deterioration detection and related clinical outcomes in hospitalised patients were included. Deterioration related outcomes (primary) included unplanned intensive care admissions, rapid response team or cardiac arrest activation, total and major complications rate. Other clinical outcomes (secondary) included in-hospital mortality and hospital length of stay. Exploratory outcomes included alerting system parameters and clinical trial registry information.

Results: Of 8706 citations, 10 studies with different designs met the inclusion criteria, of which 7 were included in the meta-analyses. Overall study quality was moderate. The meta-analysis indicated that the AMS, when compared with standard care, was associated with a reduction in intensive care transfers (risk ratio, $\mathrm{RR}, 0.87 ; 95 \%$ confidence interval, $\mathrm{Cl}, 0.66$ to 1.15$)$, rapid response or cardiac arrest team activation ( $\mathrm{RR}, 0.84 ; 95 \% \mathrm{Cl} 0.69$ to 1.01$)$, total (RR, $0.77 ; 95 \% \mathrm{Cl}$ 0.44 to 1.32 ) and major (RR, $0.55 ; 95 \% \mathrm{Cl} 0.24$ to 1.30 ) complications prevalence. There was also association with reduced mortality (RR, $0.48 ; 95 \% \mathrm{Cl} 0.18$ to 1.29 ) and hospital length of stay (mean difference, $\mathrm{MD},-0.09 ; 95 \% \mathrm{Cl}-0.43$ to 0.44 ). However, none were statistically significant.

Conclusion: This systematic review indicates that implementation of AMS may have a positive impact on early deterioration detection and associated clinical outcomes, but differing design/quality of available studies and diversity of outcomes measures limits a definite conclusion. Our narrative findings suggested that alarms should be adjusted to minimise false alerts and promote rapid clinical action in response to deterioration.

\section{PROSPERO Registration number: CRD42020188633}

\section{Background}

In the United Kingdom, the use of physiological early warning scoring (EWS) systems (which measure "standard" vital signs such as pulse rate, respiratory rate, blood pressure, oxygen saturation, and temperature) is still common practice in general wards, together with a graded response such as referral for a senior review or increasing monitoring frequency [1]. This frequency of observations is generally guided by the clinical condition of the patient, and due to the requirement of manual physiological measurements, it can be time-consuming for healthcare professionals [2]. As a result, the optimal monitoring frequency is often not achieved [3], limiting the efficacy of intermittent monitoring systems dependent on the frequency of manual observations [4]. Furthermore, even when the ideal frequency is achieved, patients can deteriorate between observation sets [5]. Higher risk patients are often continuously monitored (for example in critical care), improving early detection of deterioration [2]. However, in the UK, continuous monitoring is not commonly used in the ward environment [6], although one study suggests it may be feasible and cost-effective in surgical wards [7], with the potential to improve patient outcomes when compared to intermittent monitoring [5].

Despite the potential to promote earlier detection of deterioration, limitations in continuous vital sign monitoring technology can pose a barrier to implementation [2], such as restriction of patient mobility and independence due to wires and static devices [6, 8]. In response to this need, commercially available wearable monitoring devices are evolving rapidly [9]. Wearable devices may provide an alternative to static wired continuous monitors and offer a bridge between bedside wired monitoring and intermittent manual measurements. This development has the potential to promote patients' mobility and comfort while reducing nursing time and improving the early detection of abnormal physiological parameters [10].

A recent meta-analysis assessed the impact of multi-parameter continuous non-invasive monitoring in hospital wards, including wired static devices, suggesting a $39 \%$ decreased mortality risk in monitored patients compared to those receiving standard care (intermittent manual observations), it also suggested a trend of reduced intensive care unit (ICU) transfer, rapid response team (RRT) activation and hospital length of stay (LoS) [11]. The validation, feasibility, costs and clinical outcomes of 13 different wearable devices were assessed in another systematic review, which demonstrated that the majority of studies were still at the validation and feasibility phases [12], emphasising the lack of evidence assessing the impact on economic and clinical outcomes as there is still uncertainty around the impact of ambulatory monitoring systems (AMS) in the hospital environment, hindering its implementation and clinical use [13]. Our review focused on these ambulatory monitoring devices and/or systems implemented inside the hospital (inclusive of all specialities, acuity and ages).

\section{Objective}

The objective of this systematic review and meta-analysis was to assess the impact of vital sign monitoring on the detection of physiological deterioration and related clinical outcomes of hospitalised patients using ambulatory monitoring systems in comparison with standard care.

\section{Methods}

This systematic review was registered with the International Prospective Register of Systematic Reviews (PROSPERO) on the 10th July 2020, registration number CRD42020188633 [14]. This review was reported following the Preferred Reporting Items for Systematic Reviews and Meta-Analyses (PRISMA)

Page 2/18 
checklist (Appendix 1) [15]. The full systematic review protocol was published prospectively [16].

Outcomes

Primary Outcomes

This study aimed to compare the impact of ambulatory monitoring systems on deterioration detection and related clinical outcomes metrics, in comparison with standard care. A variety of outcomes related to deterioration detection were anticipated, and therefore searches were not limited by outcome. Any outcome related to the detection of deterioration was included as a primary outcome for this review. Specific outcomes reported in the included studies were: intensive care unit (ICU) transfers; and rapid response team activation or cardiac calls...

A variety of other complications related to clinical deterioration were reported and included in the meta-analysis, from minor (for example fainting, or shortness of breath) to major (such as life-threatening events). A separate analysis was then conducted for the studies separately reporting major complications; the Clavien-Dindo system [17] was applied to postoperative complications in the included studies. This system grades complications from I (deviation from usual recovery not requiring intervention) to $\mathrm{V}$ (patient death). To be included in our major complication meta-analysis we included complications defined by a Clavien-Dindo grade of $>$ II $(8,18)$. Patient death and ICU transfer were not included in this analysis and were assessed separately.

Outcomes reported in less than 3 studies were not included in the meta-analysis and were instead narratively described such as time to antibiotic administration in case of sepsis and number of the National Early Warning Score (NEWS) measurements.

Secondary Outcomes

Secondary outcomes in the meta-analysis included in-hospital mortality and hospital length of stay. Further secondary patient outcomes were reported in the narrative analysis, such as 30-day readmission rates and time to post-operative mobilisation

Exploratory Outcomes

Exploratory outcomes included the alerting systems used, implementation and iterations in clinical practice. This included type of early warning score, alert thresholds used for each vital sign or overall EWS and other relevant alert parameters/information, where available.

Clinical trial registry searches were also conducted. For included studies that were registered, a comparison was made between the details in registration and report of the study. Registered studies eligible for inclusion but without published results were also narratively reported.

Eligibility criteria

\section{Population and interventions}

Complete inclusion and exclusion criteria are available in the published protocol [16]. We included any studies conducted in hospitalised patients, excluding studies conducted in healthy volunteers or non-hospitalised patients.

Studies were eligible for inclusion if they used an ambulatory monitoring system (with or without standard care) in comparison with standard care. An ambulatory monitoring system was defined as a wearable system monitoring at least one vital sign (heart rate, respiratory rate, temperature, blood pressure or oxygen saturation) sampled continuously at a high rate (e.g. under a minute) or low rate (e.g. every 5 minutes) and where measurements did not require frequent manual input from clinical staff. For comparator we considered any type of standard care for vital sign monitoring, as defined in the protocol [16]. Study types

Studies with the following designs were considered for inclusion: randomised controlled trials (RCTs), cluster RCTs, interventional studies, observational studies (including case-control and before-after studies), and pilot studies. Retrospective studies that complied with the proposed outcomes and eligibility criteria, and unpublished (grey) literature, were also considered.

Literature Search and Selection of Studies

Search terms were designed by a medical librarian with field expertise (NT). Relevant articles up to 27th August 2020 were identified through electronic searches on MEDLINE Ovid (including Epub Ahead of Print and In-Process \& Other Non-Indexed Citations), Embase (Ovid), CINAHL (EBSCO) and Cochrane Database of Systematic Reviews (Cochrane Library, Wiley), Cochrane Central Register of Controlled Trials (CENTRAL) (Cochrane Library, Wiley) and Health Technology Assessment (HTA) database via https://www.crd.york.ac.uk/CRDWeb/. Full search strategy and terms/combinations used in each database can be found in Appendix 2.

Clinical trials and prospective studies registered up to 10th September 2020 in ClinicalTrials.gov via https://clinicaltrials.gov/ and ISRCTN via https://www.isrctn.com/ were also identified. Search details in Appendix 3.

Search of unpublished grey literature and pre-print servers was also conducted manually (search details in Appendix 4) and additional studies published in these servers up to 16th December 2020 were identified.

Titles and abstracts of all potentially relevant articles were independently reviewed for possible inclusion by two authors (CA, CB). The full text of any citation considered potentially relevant by any reviewer was retrieved. The degree of interrater agreement for study selection was determined by using kappa, with standard definitions for poor $(<0.20)$, fair $(0.21$ to 0.40$)$, moderate $(0.41$ to 0.60$)$, good $(0.61$ to 0.80$)$, and very good $(0.81$ to 1.00$)$ agreement $[18,19]$. The

Page $3 / 18$ 
included abstracts full-texts were assessed for eligibility and disagreements resolved by discussion between the 2 review authors; if no agreement could be reached, a third author was consulted (SV). The full selection process is outlined in the published protocol [16].

Data collection and extraction

Two reviewers (CA and CB) extracted the data independently from the included studies. Disagreements were resolved by discussion between the 2 review authors. When required, this was also discussed with a third author (MS) and a statistician (SG).

The following data were extracted for each study, where available: author list, country, date published, registration number, aim, design, setting and population, recruitment start and end dates, ethical approval and informed consent information, eligibility criteria, intervention description, included devices, period of device wear, vital signs measured by devices, frequency of ambulatory data availability, comparator type, EWS and frequency of manual measurements, sample size, demographics (e.g. age, gender, BMI, etc.), other clinical characteristics (e.g. type of admission, American Society of Anaesthesiologists, ASA, score, etc.), deterioration detection and related clinical outcomes summary data, total/median monitoring hours, alerting system information (e.g. thresholds and alarms description), study limitations, device FDA/CE mark information, funding and conflict of interest information.

Risk of Bias of Individual Studies

Four tools, selected based on study design, were used to assess risk of bias. For randomised controlled trials the Cochrane risk of bias tool (RoB2) was used [20]; for non-randomised studies, the Newcastle Ottawa Scale (NOS) [21] and the "Risk Of Bias In Non-randomised Studies - of Interventions" (ROBINS-I) were applied [22]; and, in addition, the Mixed Methods Appraisal tool (MMAT) [23] was used for all studies. This was a change from the original protocol [16] as the Jadad scale was replaced by the ROBINS-I for assessment of non-randomised studies, as we found it more comparable with the ROB2 tool used for included RCTs. Two reviewers (CA and CB) independently appraised each study and disagreements were solved by discussion until consensus was reached with a third reviewer (SV).

Data Analysis

All outcomes with results from at least three studies were considered for the meta-analysis. Outcomes with data from less than three studies were not included in the meta-analysis but reported in a narrative synthesis.

Data preparation and meta-analysis

Review Manager 5.4.1 (The Cochrane Collaboration, Oxford, England [24]) was used to calculate pooled risk ratios (RRs) for dichotomous outcomes and pooled weighted mean differences (WMDs) for continuous outcomes, and respective $95 \%$ confidence intervals (Cls). Continuous variables are expressed as mean (SD). Due to differences in design between included studies, we used random-effects meta-analysis and the TAU2 statistic, and respective significance level was calculated [25]. We assessed heterogeneity among trials by using $\mathrm{I}^{2}$ (the percentage of total variability across studies attributable to heterogeneity rather than to chance) and used published guidelines for interpretation [24].

One before-and-after study compared the AMS group with a before period in the same unit and a different unit (both before and during) [26]. For the metaanalysis, we limited data to that reported from the same unit to minimising confounding. Outcomes for this study were also presented per 1000 discharges. As the authors provided the total number of discharges, the actual event numbers were calculated for inclusion in our analysis [26]. Similarly, another included study presented the hospital length of stay (LoS) in hours [27], this was converted to days for the analysis. In a further study, the authors presented LoS in median (Interquartile range, IQR), which was converted to mean (standard deviation, SD). A normal distribution of the values was assumed to make this conversion, as per Cochrane guidance [28].

Finally, in one study complication data was presented as the number of events rather than the number of patients suffering a complication [29, 30]. A formal data request to the principal investigator was made to acquire the data in the correct format, and this was used in the meta-analysis.

Narrative analysis

Alerting thresholds, methods and other alarm information was extracted from the included studies, where available, and narratively reported. For analysis of study registration, the proportion of registered studies that were published, and both the dates of trial registration and publication of results were reported. We also explored registered versus published primary and secondary outcomes. Principal Investigators for the included and registered studies were contacted for further information, as required.

Body of evidence summary

A body of evidence summary is provided in Appendix 5, using the GRADEpro software [31].

\section{Results}

Study selection

After removal of duplicates, 8706 studies were identified. Following title and abstract review 51 full texts remained, of which 10 met the inclusion criteria (Fig. 1). Four studies appeared to meet the inclusion criteria but were excluded at full-text review: two studies were excluded for not reporting a subset of their analysis for the patients using the AMS [32,33]; one was excluded after confirming with the author that the device was not ambulatory at the time of the study [34], and another did not have a comparator group [35]. A total of 4433 patients were included in these studies.

Study Characteristics

Page 4/18 
Devices

A variety of devices were used in the studies included in this review: four studies used the VisiMobile (Sotera Visi Mobile, San Diego, California) [26, 36-38], with two studies also adding the HealthPatch (Vital Connect, Campbell, CA, USA) [37, 38]; two studies used Sensium Vitals (Sensium, Abingdon, United Kingdom) [29, 30]. The remaining four studies used different devices, including the Patient Status Engine (Isansys Lifecare Ltd.) [27], the Auricall monitoring system [39], the Avant-4100 (Nonin) [40] and the Monica Novii wireless patch system (General Electric Company, Milwaukee, WI) [41].

Included studies and outcomes

Table 1

Characteristics of the included studies. AMS: ambulatory monitoring system, BP: blood pressure, CEWS: centile-based early warning score, ESS: efficacy sa score, EWS: early warning score, HR: heart rate, NEWS: national early warning score, PSE: Patient Status Engine, RR: respiratory rate, SpO2: peripheral oxyg saturation, T: temperature. *Same patients. Weenk et al. 2020 added 30 controls. ** Both maternal and fetal heart rate

\begin{tabular}{|c|c|c|c|c|c|c|c|c|c|c|c|c|c|c|}
\hline \multirow[t]{2}{*}{ Source } & \multirow[t]{2}{*}{ Country } & \multirow[t]{2}{*}{ Population } & \multirow{2}{*}{$\begin{array}{l}\text { Sample } \\
\text { size }\end{array}$} & \multicolumn{2}{|l|}{ Interventions } & \multicolumn{5}{|c|}{ Vital signs measured by AMS } & \multicolumn{2}{|c|}{ Age (years) } & \multicolumn{2}{|c|}{ Sex (\% male } \\
\hline & & & & $\begin{array}{l}\text { Control } \\
\text { (EWS) }\end{array}$ & AMS & HR & RR & SpO2 & BP & $\mathbf{T}$ & Control & AMS & Control & A \\
\hline \multicolumn{15}{|c|}{ Meta-analysis } \\
\hline $\begin{array}{l}\text { Skraastad } \\
\text { et al. } 2019\end{array}$ & Norway & $\begin{array}{l}\text { General } \\
\text { surgery }\end{array}$ & 195 & $\begin{array}{l}\text { Intermittent } \\
\text { vital signs } \\
\text { (NEWS) }\end{array}$ & $\begin{array}{l}\text { PSE (NEWS) + } \\
\text { ESS }\end{array}$ & $x$ & $x$ & $x$ & $x$ & $x$ & 62 & 61 & $63 \%$ & 6 \\
\hline $\begin{array}{l}\text { Downey et } \\
\text { al. } 2020\end{array}$ & UK & $\begin{array}{l}\text { General } \\
\text { surgery }\end{array}$ & 135 & $\begin{array}{l}\text { Intermittent } \\
\text { vital signs } \\
\text { (NEWS) }\end{array}$ & $\begin{array}{l}\text { SensiumVitals } \\
+ \text { NEWS }\end{array}$ & $x$ & $x$ & & & $x$ & 62 & 65 & $54 \%$ & 5 \\
\hline $\begin{array}{l}\text { Downey et } \\
\text { al. } 2018\end{array}$ & UK & $\begin{array}{l}\text { General } \\
\text { surgery }\end{array}$ & 226 & $\begin{array}{l}\text { Intermittent } \\
\text { vital signs } \\
\text { (NEWS) }\end{array}$ & $\begin{array}{l}\text { SensiumVitals } \\
+ \text { NEWS }\end{array}$ & $\mathrm{x}$ & $x$ & & & $x$ & 63.7 & 65.2 & $45 \%$ & 5 \\
\hline $\begin{array}{l}\text { Kisner et } \\
\text { al. } 2009\end{array}$ & Switzerland & $\begin{array}{l}\text { General } \\
\text { surgery }\end{array}$ & 357 & $\begin{array}{l}\text { Intermittent } \\
\text { vital signs } \\
\text { (Unknown) }\end{array}$ & Auricall & $\mathrm{x}$ & & $\mathrm{x}$ & & & 62.7 & 65 & $75 \%$ & 7 \\
\hline $\begin{array}{l}\text { Weller et } \\
\text { al. } 2018\end{array}$ & USA & $\begin{array}{l}\text { Neurology } \\
\text { and } \\
\text { neurosurgery }\end{array}$ & 1958 & $\begin{array}{l}\text { Intermittent } \\
\text { vital signs } \\
\text { (Unknown) }\end{array}$ & VisiMobile & $x$ & $x$ & $x$ & $\mathrm{x}$ & $\mathrm{x}$ & 59.3 & 60.5 & $58 \%$ & 5 \\
\hline $\begin{array}{l}\text { Verrillo et } \\
\text { al. } 2018\end{array}$ & USA & $\begin{array}{l}\text { Orthopaedic, } \\
\text { spinal and } \\
\text { trauma } \\
\text { general care }\end{array}$ & 849 & $\begin{array}{l}\text { Intermittent } \\
\text { vital signs } \\
\text { (Unknown) }\end{array}$ & VisiMobile & $x$ & $x$ & $x$ & $x$ & $x$ & 51.4 & 54.5 & $58 \%$ & 5 \\
\hline $\begin{array}{l}\text { Watkinson } \\
\text { et al. } 2020\end{array}$ & UK & $\begin{array}{l}\text { Gastro } \\
\text { intestinal } \\
\text { surgery }\end{array}$ & 407 & $\begin{array}{l}\text { Intermittent } \\
\text { vital signs } \\
\text { (CEWS) }\end{array}$ & Avant-4100 & $\mathrm{x}$ & & $x$ & & & 63 & 63 & $56 \%$ & 5 \\
\hline \multicolumn{15}{|l|}{ Narrative } \\
\hline \multirow[t]{2}{*}{$\begin{array}{l}\text { Weenk et } \\
\text { al. } 2019\end{array}$} & \multirow[t]{2}{*}{ Netherlands } & \multirow{2}{*}{$\begin{array}{l}\text { Surgical and } \\
\text { internal } \\
\text { medicine } \\
\text { ward }\end{array}$} & \multirow[t]{2}{*}{$60 *$} & \multirow{2}{*}{$\begin{array}{l}\text { Intermittent } \\
\text { measurement } \\
\text { (MEWS) }\end{array}$} & VisiMobile & $\mathrm{x}$ & $\mathrm{x}$ & $\mathrm{x}$ & $\mathrm{x}$ & $x$ & $\mathrm{~N} / \mathrm{A}$ & 63 & $\mathrm{~N} / \mathrm{A}$ & 6 \\
\hline & & & & & HealthPatch & $x$ & $x$ & & & $x$ & N/A & 56 & N/A & 7 \\
\hline \multirow[t]{2}{*}{$\begin{array}{l}\text { Weenk et } \\
\text { al. } 2020\end{array}$} & \multirow[t]{2}{*}{ Netherlands } & \multirow{2}{*}{$\begin{array}{l}\text { Surgical and } \\
\text { internal } \\
\text { medicine } \\
\text { ward }\end{array}$} & \multirow[t]{2}{*}{$90 *$} & \multirow{2}{*}{$\begin{array}{l}\text { Intermittent } \\
\text { measurement } \\
\text { (MEWS) }\end{array}$} & VisiMobile & $\mathrm{x}$ & $x$ & $x$ & $x$ & $x$ & 62 & 63 & $67 \%$ & 6 \\
\hline & & & & & HealthPatch & $x$ & $x$ & & & $x$ & & 56 & & 7 \\
\hline \multirow{2}{*}{$\begin{array}{l}\text { Monson et } \\
\text { al. } 2020\end{array}$} & \multirow[t]{2}{*}{ USA } & \multirow{2}{*}{$\begin{array}{l}\text { Labour and } \\
\text { delivery } \\
\text { ward }\end{array}$} & \multirow[t]{2}{*}{216} & \multirow{2}{*}{$\begin{array}{l}\text { Standard } \\
\text { external } \\
\text { monitoring }\end{array}$} & Monica & $x^{\star \star}$ & & & & & 28.6 & 29.2 & $0 \%$ & 0 \\
\hline & & & & & Novii & & & & & & & & & \\
\hline
\end{tabular}

Of the ten studies identified, seven were included in the meta-analysis with a total of 4127 patients. These included two RCTs [27, 30], one cluster RCT [29], and four before-and-after observational studies [26, 36, 39, 40]. Three further studies (RCTs) were included in the narrative synthesis [37, 38, 41], including a total of 306 further patients. Details of the included studies are presented in Tables 1 and 2.

The majority of the included studies implemented the AMS in post-surgical patients. Four studies also reported the patient American Society of Anaesthesiologists (ASA) score for preoperative functional status [42], with a median ASA score of 2 ("Patient has mild systemic disease") in three studies [29, 30,43 ] and 3 ("Patient has severe systemic disease that is not incapacitating.") in one [27].

Reviewers achieved a fair level of agreement (kappa: 0.348 ; $95 \% \mathrm{Cl} 0.285$ to 0.482 ) for study inclusion. There were no major disagreements between reviewers regarding data extraction, study quality or bias assessments. 
Table 2

Included studies primary outcomes and outcomes included in the meta-analysis.

\begin{tabular}{|c|c|c|c|c|c|c|c|c|}
\hline \multirow[t]{2}{*}{ Source } & \multirow[t]{2}{*}{ Design } & \multirow[t]{2}{*}{ Primary outcome } & \multicolumn{4}{|c|}{ Deterioration detection outcomes } & \multicolumn{2}{|c|}{ Clinical Outcomes } \\
\hline & & & $\begin{array}{l}\text { ICU } \\
\text { transfer }\end{array}$ & $\begin{array}{l}\text { RRT activation or } \\
\text { cardiac arrest team } \\
\text { call }\end{array}$ & $\begin{array}{l}\text { All } \\
\text { complications }\end{array}$ & $\begin{array}{l}\text { Major } \\
\text { complications }\end{array}$ & Mortality & $\begin{array}{l}\text { Length } \\
\text { of stay }\end{array}$ \\
\hline \multicolumn{9}{|c|}{ Meta-analysis } \\
\hline $\begin{array}{l}\text { Skraastad } \\
\text { et al. } 2019\end{array}$ & $\mathrm{RCT}$ & Time to mobilisation & & & $x$ & $x$ & $x$ & $x$ \\
\hline $\begin{array}{l}\text { Downey et } \\
\text { al. } 2020\end{array}$ & $\begin{array}{l}\text { Pilot } \\
\text { RCT }\end{array}$ & Progression criteria to full RCT & $x$ & & $x$ & $\mathrm{X}$ & $\mathrm{X}$ & $\mathrm{x}$ \\
\hline $\begin{array}{l}\text { Downey et } \\
\text { al. } 2018\end{array}$ & $\begin{array}{l}\text { Cluster } \\
\text { RCT }\end{array}$ & $\begin{array}{l}\text { Time to antibiotics in patients with } \\
\text { sepsis }\end{array}$ & $x$ & & $x$ & $\mathrm{x}$ & $x$ & $x$ \\
\hline $\begin{array}{l}\text { Kisner et al. } \\
2009\end{array}$ & $\begin{array}{l}\text { Before- } \\
\text { after }\end{array}$ & $\begin{array}{l}\text { Incidence of postoperative atrial } \\
\text { fibrillation }\end{array}$ & & & $\mathrm{x}$ & & & \\
\hline $\begin{array}{l}\text { Weller et al. } \\
2018\end{array}$ & $\begin{array}{l}\text { Before- } \\
\text { after }\end{array}$ & Rate of patient deterioration events & $x$ & $\mathrm{x}$ & & & $\mathrm{x}$ & $x$ \\
\hline $\begin{array}{l}\text { Verrillo et } \\
\text { al. } 2018\end{array}$ & $\begin{array}{l}\text { Before- } \\
\text { after }\end{array}$ & Early deterioration detection & $x$ & $x$ & $x$ & & $x$ & \\
\hline $\begin{array}{l}\text { Watkinson } \\
\text { et al. } 2020\end{array}$ & $\begin{array}{l}\text { Before- } \\
\text { after }\end{array}$ & Length of stay & $x$ & $x$ & & & $x$ & $x$ \\
\hline \multicolumn{9}{|l|}{ Narrative } \\
\hline $\begin{array}{l}\text { Weenk et } \\
\text { al. } 2019\end{array}$ & RCT & $\begin{array}{l}\text { Experiences of patients and care } \\
\text { givers }\end{array}$ & & & & & & \\
\hline \multicolumn{9}{|l|}{$\begin{array}{l}\text { Weenk et } \\
\text { al. } 2020\end{array}$} \\
\hline $\begin{array}{l}\text { Monson et } \\
\text { al. } 2020\end{array}$ & $\mathrm{RCT}$ & $\begin{array}{l}\text { Amount of time with the } \\
\text { interpretable fetal HR tracing } \\
\text { during of labour }\end{array}$ & & & & & & \\
\hline
\end{tabular}

Studies not included in the meta-analysis were narratively explored (Tables 1 and 2). Two papers reported results from one RCT, comparing two devices (HealthPatch and VisiMobile) with nurse measurements [37, 38]. However, they have not included the third group (control) in the analysis and not assessed any clinical outcomes, mostly exploring factors related to deterioration detection, failing to provide sufficient data to include in the meta-analysis. In the first paper from this RCT, the authors report that both HealthPatch and VisiMobile modified early warning scores (MEWS) were higher than the nurse measured MEWS, mostly due to RR measurements differences [38]. In the second paper (the full RCT) the authors identified positive and negative effects as well as barriers and facilitators for the use of these devices, such as the impact of AMS in a shorter length of stay and prevention of ICU admissions, additionally, a total of 17 patients, 2 relatives and 17 healthcare professionals reported to be expecting earlier deterioration detection using these wearables [37].

Another RCT evaluated wireless external fetal electrocardiography versus standard external monitoring [41]. We were unable to include this study in the metaanalysis as (1) the primary outcome of the study was the percentage of interpretable fetal HR data, (2) the population of interest is very different from the remaining studies and (3) the clinical outcomes analysed also differed (eg. length of labour, fetal Apgar score, etc.). Considering this, their results demonstrated no differences in maternal or neonatal clinical outcomes between groups. However, results did suggest an increased acceptance by patients and staff, with satisfaction scores significantly higher when compared to the standard monitor [41].

Included studies registration

Details of the clinical trials search are shown in Appendix 6. Of the ten included studies in this review, only seven were registered (most retrospectively, as per Appendix 7). Within these, all primary outcomes stated in the registration were reported in the main paper, as well as most of the secondary outcomes.

Study quality and risk of bias

The overall quality of included studies was moderate with some bias to take into account, as per Figs. 2 and 3. For the included RCTs, using the ROB2, two were identified as at "low risk" of bias [30,41] and a further three were assessed as raising "some concerns" [27, 37], including the cluster RCT [29]. The risk of bias, assessed by the ROBINS-I was "moderate" for all before-and-after studies [26, 36, 39, 40]. See Appendix 8 for further details. The results of the bias assessment did not influence inclusion in the meta-analyses.

Primary outcomes

In total, data from seven studies were included in the meta-analysis of primary outcomes related to deterioration detection, analysed separately according to the three reported deterioration outcomes - ICU transfers, rapid response or cardiac arrest activation, and complications.

ICU transfers 
A total of five studies reported ICU transfers and were included in this meta-analysis (data from 3565 patients, 1898 in the AMS group) [26, 29, 30, 36, 40].

Pooling of data indicated that use of AMS did reduce ICU transfer (RR, $0.87 ; 95 \% \mathrm{Cl} 0.66$ to 1.15$)$, but not statistically significantly ( $p=0.32)$ (Fig. 4).

Rapid response or cardiac arrest activation

For this outcome, two before-and-after studies reporting rapid response team activation and another study reporting cardiac arrest calls were included (with data from 3214 patients, 1698 in the AMS group, Fig. 5) [26, 36, 40]. Pooled data for this outcome indicated AMS reduced RRT or cardiac arrest calls (RR, 0.84; $95 \% \mathrm{Cl} 0.69$ to 1.01$)$ with a $\mathrm{p}$-value near statistical significance $(\mathrm{p}=0.07)$.

All clinical complications

A total of five studies reported data on complication outcomes classed by the Clavien-Dindo system as grade I or II(with data from 1752 patients, 837 in the AMS group, Fig. 6). indicating the AMS group had a reduced risk of complications (RR, $0.77 ; 95 \% \mathrm{Cl} 0.44$ to 1.32$)$ however without statistical significance $(\mathrm{p}=$ 0.34) and with high heterogeneity between studies $\left(I^{2}=93 \%\right)$.

For the major complications (Fig. 7), we included 3 studies (with data from 546 patients, 296 in the AMS group) indicating the AMS group had reduced risk of major complications (RR, $0.55 ; 95 \% \mathrm{Cl} 0.24$ to 1.30 ) however, with no statistical significance $(\mathrm{p}=0.17)$.

Other deterioration detection outcomes not included in the meta-analysis

A few of the included studies also explored other deterioration detection outcomes, but in insufficient numbers to allow a meta-analysis. One cluster RCT [29] and one RCT [30] from the same research group compared the time to antibiotic administration in case of sepsis in the AMS group against the control group, finding this statistically insignificant in both studies $(656.0$ (95\% Cl 431.7-820.3) vs 1012.8 (95\% $\mathrm{Cl} 425.0-1600.6)$ minutes [29] and 551 (95\% Cl 296-805) vs $527(95 \%$ Cl 199-856)) [30].

Secondary outcomes

The two secondary outcomes of in-hospital mortality and hospital length of stay were also meta-analysed. In-hospital mortality

For the outcome of in-hospital mortality, we included six studies (with data from 3760 patients, 1994 in the AMS group, Fig. 8) [26, 27, 29, 30, 36, 40], with one study reporting no deaths in either group (no estimates to be analysed in the meta-analysis) [27]. Our results indicated the AMS group had a reduced risk of mortality (RR, $0.48 ; 95 \% \mathrm{Cl} 0.18$ to 1.29$)$ but this reduction was not statistically significant $(\mathrm{p}=0.15)$.

Hospital length of stay

A total of five studies were included for the outcome of hospital LoS (with data from 2911 patients, 1994 in the AMS group, Fig. 9) indicating a non-significant reduction in hospital length of stay for patients monitored using AMS (MD, $-0.09 ; 95 \% \mathrm{Cl}-0.43$ to $0.44, \mathrm{p}=0.63)$.

Other clinical outcomes not included in the meta-analysis

Studies also included other clinical outcomes, for example, two studies explored 30-day hospital readmission rates and showed mixed results, with one showing lower readmission rates in the AMS group [29] and the other slightly higher [30] in comparison with standard care.

Skraastad and colleagues indicated reduced time to post-operative mobilisation in the AMS group, 10.1 (95\% $\mathrm{Cl} 8.1-12.2)$ against 14.2 (95\% $\mathrm{Cl} 12.0-16.3)$ in the control group [27]. They also compared the number of NEWS measurements in their RCT, with 8.2 (95\% $\mathrm{Cl} 47.4-9.0)$ in the AMS group versus 3.4 (95\% Cl 3.1-3.6) in the standard care group. Additionally, there was a higher mean in opioid dose given in the AMS group, 25.5 ( $95 \% \mathrm{Cl} 20.9-30.0)$ vs 15.2 ( $95 \% \mathrm{Cl}$ $11.1-19.3$ ) in the control arm; and more supplementary oxygen was given to 57/96 in the AMS group against 32/99 in the control group [27], that the authors defend being a result of the increased monitoring in the AMS group, facilitating pain and oxygen management of those patients, and promoting earlier mobilisation [27]. This study also

\section{Exploratory outcomes}

Alerting systems (Central station and mobile devices for alerts)

Some information about the alerting system was available in nine out of the ten included studies [26, 27, 29, 30, 36, 38-40, 44]. Five studies reported their development in reducing the number of alarms per patient per day (APDs). One reported having started with 11.41 APDs and conducted iterations down to 2.01 APDs, reducing the non-actionable alarms and modifying their vital sign limits and thresholds to reduce the rate of false alarms [26]. The authors focused on monitoring optimisation, reviewing and modelling the alarm data every few days and discussing with clinical managers whether widening vital sign parameters would create a significant reduction in alarm rates while still being clinically acceptable and sensible to deterioration detection [26]. In Downey and colleagues' first study there was an unacceptable number of alerts sent to the nursing staff. After adjustments in the vital sign thresholds, the false alerts were reduced by $90 \%$ (30). This was addressed in their second RCT where a clinical fellow was visiting the wards daily to check the rate of false alarms and adjust thresholds and/or delays of the alerts as per clinical need $(18,48)$. Despite this, two patients withdrew from the study due to "too many false alerts" $(18)$. In two other studies, the authors just discussed the intention was to improve the rate of true positives and reduce false negatives/false alarms [37, 38].

Most alerting thresholds were pre-set and individualised as required, alerted through the central station and/or nurse mobile/pager/PDA, with the majority using audio alerts (Table 4). In one study the authors used a single risk score calculated from all vital signs (VSI) and based on modelling from a previous patient dataset, creating an alert when the VSI score was above the threshold for more than 4 out of 5 minutes [40]. Alerting parameters from the included studies are explored in Table 4. 
In one study's final version of the alerting system, hypotension, bradycardia and hypoxemia were tolerated for shorter periods than tachycardia or hypertension, unless the tachycardia resulted in hypotension. Additionally, the majority of the alerts in this final iteration were due to Sp02 (0.97 APDs) [26]. Another study found that the most accurate vital sign parameter was systolic blood pressure, which had a positive predictive value (PPV) of $97 \%$, followed by high respiratory rate (PPV of $85 \%$ ) and low $\mathrm{SpO}_{2}$ (PPV of 76\%), indicating high sensitivity and reliability and a low false alarm rate. [36]

Table 3

Vital sign thresholds summary table. BP: blood pressure, DBP: diastolic blood pressure, HR: heart rate, MAP: mean arterial pressure, PDA: personal digital assistant, PR: pulse rate, RR: respiratory rate, SBP: systolic blood pressure, SMS: short message service, Sp02: peripheral oxygen saturation, T: temperature, VSI: Visensia Safety Index.

\begin{tabular}{|c|c|c|c|c|c|c|c|c|c|c|}
\hline \multirow{3}{*}{ Study } & \multicolumn{9}{|c|}{ Vital sign thresholds (delay before alert in seconds) } & \multirow[t]{3}{*}{ Alert method } \\
\hline & \multicolumn{2}{|l|}{ HR/PR } & \multicolumn{2}{|l|}{ RR } & \multirow{2}{*}{$\begin{array}{l}\text { SpO2 } \\
\text { Low }\end{array}$} & \multicolumn{2}{|l|}{ BP } & \multirow{2}{*}{$\begin{array}{l}\text { MAP } \\
\text { Low }\end{array}$} & \multirow[t]{2}{*}{$\mathbf{T}$} & \\
\hline & High & Low & High & Low & & $\begin{array}{l}\text { High } \\
\text { SBP }\end{array}$ & $\begin{array}{l}\text { Low } \\
\text { DBP }\end{array}$ & & & \\
\hline \multirow{3}{*}{$\begin{array}{l}\text { Weller et al. } \\
2018\end{array}$} & \multirow{2}{*}{$\begin{array}{l}\text { HR: } \\
150 \\
(15)\end{array}$} & HR: 39 (15) & \multirow{3}{*}{$\begin{array}{l}35 \\
(120)\end{array}$} & \multirow[t]{3}{*}{$4(120)$} & \multirow[t]{3}{*}{$85(90)$} & \multirow{3}{*}{\multicolumn{2}{|c|}{$\begin{array}{l}200 \\
(240)\end{array}$}} & \multirow{3}{*}{$\begin{array}{l}58 \\
(60)\end{array}$} & & Sound \\
\hline & & PR: 39 (60) & & & & & & & & $\begin{array}{l}\text { Central station } \\
+ \text { nurse phone }\end{array}$ \\
\hline & \multicolumn{2}{|l|}{$\begin{array}{l}\text { PR: } \\
150 \\
(60)\end{array}$} & & & & & & & & \\
\hline \multirow{2}{*}{$\begin{array}{l}\text { Kisner et al. } \\
2009\end{array}$} & \multirow{2}{*}{\multicolumn{2}{|c|}{$\begin{array}{l}\text { Individualised } \\
\text { (immediate) }\end{array}$}} & & & \multirow{2}{*}{$\begin{array}{l}\text { Pre-set } \\
\text { individualised } \\
\text { (immediate) }\end{array}$} & & & & & Sound \\
\hline & & & & & & & & & & $\begin{array}{l}\text { Pager or SMS } \\
\text { to doctor and } \\
\text { nurse }\end{array}$ \\
\hline \multirow{2}{*}{$\begin{array}{l}\text { Verrilo et al. } \\
2018\end{array}$} & \multirow{2}{*}{\multicolumn{2}{|c|}{ Pre-set (5) }} & \multirow{2}{*}{\multicolumn{2}{|c|}{ Pre-set (120) }} & \multirow[t]{2}{*}{ Pre-set (60) } & \multirow{2}{*}{\multicolumn{2}{|c|}{ Pre-set (120) }} & & & Sound \\
\hline & & & & & & & & & & $\begin{array}{l}\text { Central } \\
\text { monitor + } \\
\text { nurse mobile }\end{array}$ \\
\hline \multirow{2}{*}{$\begin{array}{l}\text { Downey et } \\
\text { al. } 2018\end{array}$} & \multirow{2}{*}{\multicolumn{2}{|c|}{$\begin{array}{l}\text { Pre-set, individualised } \\
\text { if required } \\
\text { (immediate) }\end{array}$}} & \multirow{2}{*}{\multicolumn{2}{|c|}{$\begin{array}{l}\text { Pre-set, individualised if } \\
\text { required (immediate) }\end{array}$}} & & & & & $\begin{array}{l}\text { Pre-set, } \\
\text { individualised if }\end{array}$ & Sound \\
\hline & & & & & & & & & $\begin{array}{l}\text { required } \\
\text { (immediate) }\end{array}$ & $\begin{array}{l}\text { Mobile device } \\
\text { to nurse }\end{array}$ \\
\hline \multirow[t]{2}{*}{$\begin{array}{l}\text { Downey et } \\
\text { al. } 2020\end{array}$} & \multirow{2}{*}{\multicolumn{2}{|c|}{$\begin{array}{l}\text { Pre-set, individualised } \\
\text { if required } \\
\text { (immediate) }\end{array}$}} & \multirow{2}{*}{\multicolumn{2}{|c|}{$\begin{array}{l}\text { Pre-set, individualised if } \\
\text { required (immediate) }\end{array}$}} & & & & & $\begin{array}{l}\text { Pre-set, } \\
\text { individualised if }\end{array}$ & \\
\hline & & & & & & & & & $\begin{array}{l}\text { required } \\
\text { (immediate) }\end{array}$ & $\begin{array}{l}\text { Mobile device } \\
\text { to nurse }\end{array}$ \\
\hline \multirow{2}{*}{$\begin{array}{l}\text { Weenk et } \\
\text { al. } 2019 \& \\
2020\end{array}$} & \multirow{2}{*}{\multicolumn{2}{|c|}{$\begin{array}{l}\text { Individualised } \\
\text { (immediate) }\end{array}$}} & Individu & lised (immediate) & $\begin{array}{l}\text { Individualised } \\
\text { (immediate) }\end{array}$ & $\begin{array}{l}\text { Individ } \\
\text { (imme }\end{array}$ & $\begin{array}{l}\text { alised } \\
\text { iate) }\end{array}$ & & $\begin{array}{l}\text { Individualised } \\
\text { (immediate) }\end{array}$ & Sound \\
\hline & & & & & & & & & & Nurse station \\
\hline Skraastad & As per $\mathrm{I}$ & EWS score & As per $\mathrm{I}$ & EWS score & As per NEWS & As per & JEWS & & As per NEWS & Visual only \\
\hline & & & & & & & & & & $\begin{array}{l}\text { Warnings at } \\
\text { patient bedside }\end{array}$ \\
\hline & & Alerting score & resholds & & & & & & & \\
\hline & $\begin{array}{l}\text { Score } \\
\text { name }\end{array}$ & $\begin{array}{l}\text { Vital signs } \\
\text { included in } \\
\text { score }\end{array}$ & Range & Threshold & Time to alert & & & & & \\
\hline $\begin{array}{l}\text { Watkinson } \\
\text { et al. } 2020\end{array}$ & VSI & All & 0 to 5 & $\begin{array}{l}>3.0 \text { for more } \\
\text { than } 4 \text { out of } 5\end{array}$ & $\begin{array}{l}\text { On the fifth } \\
\text { minute }\end{array}$ & & & & & Sound \\
\hline & & & & minutes & & & & & & $\begin{array}{l}\text { Bedside + } \\
\text { central station } \\
+ \text { nurse PDA }\end{array}$ \\
\hline
\end{tabular}

Clinical trial registries (other potentially eligible studies to be included)

A total of sixteen registrations were identified in the search and screened for eligibility. Six were excluded and six registrations refer to the included studies. A further four registrations were deemed potentially eligible to be included in our review and meta-analysis (Table 3). A registered cluster RCT [45] aimed to develop a two-tiered monitoring system to improve the care of patients at risk of clinical deterioration in general hospital wards. This registered study also included a subset of patients using wireless devices [45]. However, although the main results are published [32], no data were reported on the impact of wireless devices on the subset population and we were unable to make contact with the Principal Investigator to clarify publication status and request this subset of the AMS group data. A further registration [46] before-and-after study was potentially eligible and although the main results are published [33] a subset of patients (278) used at least one cableless sensor, but the author confirmed there were no data available on outcomes for this sub-set of wirelessly monitored patients [33]. The other two registrations did not publish their results at the time of our systematic literature search. The Principal Investigator of 
one prospective, observational cohort study confirmed the study results have been submitted and is under peer review [47]. We were unable to contact the Principal Investigator to clarify the status of the other study [48].

Table 4

Studies with potential for inclusion.

\begin{tabular}{|lllll|}
\hline $\begin{array}{l}\text { Year } \\
\text { registered }\end{array}$ & Registration ID & $\begin{array}{l}\text { Date start } \\
\text { recruitment }\end{array}$ & $\begin{array}{l}\text { Recruitment } \\
\text { status }\end{array}$ & \begin{tabular}{l} 
Reason for non-publication \\
\hline 2011
\end{tabular} \\
\hline $\begin{array}{l}\text { NCT01280942 } \\
{[45]}\end{array}$ & January 2011 & Completed & $\begin{array}{l}\text { Main results published but not sub-group analysis with the wireless } \\
\text { device. [32] }\end{array}$ \\
\hline 2012 & $\begin{array}{l}\text { NCT01692847 } \\
{[46]}\end{array}$ & October 2012 & Completed & $\begin{array}{l}\text { Main results published but not sub-group analysis with the cableless } \\
\text { device. [33] }\end{array}$ \\
\hline 2017 & $\begin{array}{l}\text { NCT02427828 } \\
{[48]}\end{array}$ & March 2013 & Completed & Unknown \\
\hline & $\begin{array}{l}\text { NCT03179267 } \\
{[47]}\end{array}$ & September 2017 & Completed & Under peer review \\
\hline
\end{tabular}

\section{Discussion}

Main results

In this systematic review and meta-analysis, we identified 10 eligible studies of various designs comparing the impact of AMS on deterioration detection and clinical outcomes with standard care, including a total of 4433 patients. Our main findings indicate that AMS may have the potential to reduce ICU admissions, RRT/cardiac arrest calls and complications in hospitalised patients, although without significant significance. The strength of these findings is limited by the small number of studies, limited sample sizes, and moderate risk of bias, failing to provide a generalisable answer to our research question (GradePro summary in Appendix 5). This review also provides some limited indication that AMS may reduce in-hospital mortality and length of stay, but again without strong statistical significance supporting these findings.

Although our review focused specifically on ambulatory monitoring devices, our results are in accordance with a previous systematic review with a focus on the clinical impact of a broader range of multi-parameter continuous non-invasive monitoring of vital signs in non-intensive care unit patients. In this previous review, the authors included all non-invasive devices (including wired and static bed monitors such as EarlySense) and also found a trend towards reduced ICU transfers, RRT activations and hospital length of stay. It also suggested reduced hospital mortality in patients using these devices [11]. Our study also updates their review with the inclusion of an additional four more recent studies.

All the studies included in this review were conducted in a non-ICU environment (mostly in surgical patients) and most comparators were standard intermittent vital sign monitoring with the use of local EWS. Previous evidence suggests that focus on non-critical care settings is due to AMS being unable to replace the continuous monitoring commonly used for high dependency patients. Instead, AMS offers an intermediate level of monitoring between continuous high dependency monitoring and intermittent manual measurements, with the potential to facilitate early deterioration detection in high-risk patients (e.g. post-ICU) [2]. In addition, a recent study in the paediatric population concluded that wireless monitoring is feasible and can identify more deteriorations. The authors suggest that by using this in combination with a paediatrics early warning (PEW), some life-threatening events may be prevented [35].

One study included in this review reported patients', relatives' and healthcare professionals' perceptions of the use of AMS in the general ward, and found agreement between all interviewed groups that AMS could facilitate earlier deterioration detection and improve patient safety without posing a barrier to mobility, as well as reduce staff workload and hospital costs [37], agreeing with previous evidence [49-51] and reinforcing the direction of our findings.

To better understand the used AMS, we have aggregated available information, of the alerting methods and thresholds of the included studies. As most studies used audio alerts, system iterations seemed to focus on reducing the rate of false alerts by adjusting/individualising each vital sign and/or overall score to avoid alarm fatigue from clinical staff. This has been previously discussed as an important factor for the successful deployment of monitoring technology [52-55]. The exception was one study that used visual warnings alone, resulting in increased NEWS measurements in patients using AMS without clinical staff being aware of the potential deterioration. They identified three patients for whom AMS alarmed between two intermittent measurements and who were later diagnosed with a pneumonia, atrial fibrillation and an anastomotic leakage [38]; in the same study, the authors also explored the delay between high MEWS measured by a device and next regular MEWS measurement by a nurse, ranging from 0 up to 10 hours, and varying between day and night [38].

Finally, a review of known registries was conducted to address the issue of publication bias, finding that the majority of the included studies were registered either in ClinicalTrials.gov or ISRCTN databases and all registered primary outcomes were reported as well as most of the secondary (Appendix 7). However, most studies were registered retrospectively, rather than prospectively. We did, however, identify four studies that might have contributed to this systematic review by either performing a subgroup analysis for patients using ambulatory devices $[33,45]$ and by publishing their results as registered in another two $[47$, 48]. Our registry search allowed to highlight this under-reported and non-published evidence that could have potentially impacted our results and contributed to the meta-analysis and overall body of evidence in this field.

Study limitations

There were some limitations to this review. The number of studies included was limited and used a variety of designs, populations, outcomes, medical devices/systems, EWS and alerting thresholds. Our meta-analysis only included two RCTs (one being a pilot) and one pilot cluster RCT, all of which had small 
sample sizes which reduced the probability of significant difference in outcomes, despite large effect sizes. In contrast, the included before-and-after studies had larger sample sizes but increased bias and quality limitations, again posing a barrier to any significant conclusion. This reflects the emerging nature of this area of research and highlights the need for a large, multicentre RCT that tests whether AMS may be beneficial for early deterioration detection and related clinical outcomes in hospitalised patients.

One of the studies [29] had an extra non-randomised bay in their exploratory analysis. We did not include these data in our meta-analysis in accordance with Cochrane guidelines, so only the randomised groups were considered [56]. Similarly, another study compared the AMS group with a period before in the same unit and a period during and before in an alternative unit. In this case, we only used the data comparing the before period in the same unit, to minimise selection bias [56]. For RRT and cardiac team call analysis, it is important to note that Weller and colleagues study accounted for $96.7 \%$ of the weight on these results [26]. In addition, studies exploring RRT and cardiac team calls were combined in the meta-analysis, and therefore results might differ if analysed separately.

As previously reported, bias may be present as clinical staff is aware of the AMS use in their patients. However, the practicalities of blinding in AMS studies may not be feasible and may potentially have a counterproductive impact on clinical outcomes [11].

Future research

The results of this systematic review suggest the use of AMS might promote early deterioration detection and improve clinical outcomes. However, these findings are limited by the reduced sample size of the included RCTs and reduced methodological quality of the before-and-after studies. One of the included studies [30] conducted a subgroup analysis exploring clinical outcomes in high-risk participants. This further highlighted the impact of the AMS use in patients at higher risk of deterioration and further RCTs should consider including a similar analysis of their results.

Remote monitoring systems are only beneficial to the clinical staff if they are easy to use and clinicians understand the potential benefit for patient clinical outcomes [57]. This review is part of a wider phased project, the virtual High Dependency Unit (vHDU) study. So far, we have selected [58] and tested AMDs [59] to be integrated in a final AMS, that will be tested in a pilot and then a full multicentre RCT in the near future.

\section{Conclusions}

Our systematic review suggests that AMS may have a positive impact on early deterioration detection and associated clinical outcomes. Although no results were significant, we did find a trend towards fewer ICU admissions, RRT/cardiac arrest team calls, major and overall complications, in-hospital mortality and hospital length of stay in patients using the AMS against standard care. The included studies were limited by a reduced number of RCTs with a small sample size and before-and-after studies with moderate bias. This review highlights the need for bigger and more rigorous RCTs to support our meta-analysis findings. Additionally, our narrative findings suggest that alarms should be adjusted to minimise false alerts and thus support prompt clinical action to deterioration events.

\section{Abbreviations}

ADLs: activities of daily living

AMD: ambulatory monitoring device

AMS: ambulatory monitoring system

APD: alarm per patient per day

ASA: American Society of Anaesthesiologists

CEWS: centile-based early warning score

BP: blood pressure

Cl: confidence interval

CRS: comfort rating scale

ECG: electrocardiogram

EWS: early warning score

HR: heart rate

ICJME: international committee of medical journal editors

ICU: intensive care unit

IQR: interquartile range 
LoS: length of stay

MD: mean difference

MEWS: modified early warning score

NEWS: national early warning score

NIHR: National Institute for Health Research

NOS: Newcastle Ottawa scale

PEW: pediatric early warning

PPV: positive predictive value PR: pulse rate

RCT: Randomised Controlled Trial

RoB2: Cochrane risk of bias tool

ROBINS-I: Risk Of Bias In Non-randomised Studies - of Interventions

RR: respiratory rate

RR: risk ratio

RRT: rapid respnse team

SBP: systolic blood pressure

$\mathrm{SpO}_{2}$ : peripheral capillary oxygen saturation

UK: United Kingdom

vHDU: virtual High Dependency Unit

WMD: weighted mean difference

\section{Declarations}

\section{Ethics approval and consent to participate}

Not applicable.

\section{Consent for publication}

Not applicable

\section{Data availability statement}

All data generated or analysed during this study are included in this published article and its supplementary information files

\section{Conflicts of interest}

PW and LT report significant grants from the National Institute of Health Research (NIHR), UK and the NIHR Biomedical Research Centre, Oxford, during the conduct of the study. PW and LT report modest grants and personal fees from Sensyne Health, outside the submitted work. LT works part-time for Sensyne Health and has share options in the company. PW holds shares in the company.

\section{Funding}

The research was funded by the National Institute for Health Research (NIHR) Oxford Biomedical Research Centre (BRC). PW and LT are supported by the NIHR Biomedical Research Centre, Oxford. The views expressed are those of the author(s) and not necessarily those of the NHS, the NIHR or the Department of Health.

\section{Authors' contributions}


CA designed the study. NT conducted databases search. PW and LT made final approval of the study protocol. CA and CB selected studies for inclusion, SV resolved any disagreements. CA and CB extracted the data from studies, SG and MS reviewed data extraction. CA and CB conducted quality assessment, SV resolved disagreements. CA conducted statistical analysis with SG supervision. CA wrote the first draft of the manuscript with SV support. All authors contributed and approved the final manuscript.

\section{Acknowledgements}

We would like to acknowledge Marco Pimentel for his support in designing the research question and Louise Young for her support in the refinement of search terms definitions. We would also like to acknowledge Candice Downey and Gemma Ainsworth for sharing their TRaCINg trial data, funded by the NIHR.

\section{References}

1. NICE Clinical Guidelines. Acutely ill adults in hospital: recognising and responding to deterioration. 2007; July:1-30.

2. Prgomet M, Cardona-Morrell M, Nicholson M, Lake R, Long J, Westbrook J, et al. Vital signs monitoring on general wards: clinical staff perceptions of current practices and the planned introduction of continuous monitoring technology. Int J Qual Heal Care. 2016;28:515-21. doi:10.1093/intqhc/mzw062.

3. Clifton DA, Clifton L, Sandu DM, Smith GB, Tarassenko L, Vollam SA, et al. "Errors" and omissions in paper-based early warning scores: The association with changes in vital signs-a database analysis. BMJ Open. 2015;5:1-7.

4. Jansen JO, Cuthbertson BH. Detecting critical illness outside the ICU: the role of track and trigger systems. Curr Opin Crit Care. 2010;16:184-90. doi:10.1097/MCC.0b013e328338844e.

5. Tarassenko L, Hann A, Young D. Integrated monitoring and analysis for early warning of patient deterioration. Br J Anaesth. 2006;97:64-8.

6. Bonnici T, Tarassenko L, Clifton DA, Watkinson P. The digital patient. Clin Med. 2013;13:252-7.

7. Javanbakht M, Mashayekhi A, Trevor M, Rezaei Hemami M, L. Downey C, Branagan-Harris M, et al. Cost utility analysis of continuous and intermittent versus intermittent vital signs monitoring in patients admitted to surgical wards. J Med Econ. 2020;:1-9. doi:10.1080/13696998.2020.1747474.

8. Downey CL, Chapman S, Randell R, Brown JM, Jayne DG. The impact of continuous versus intermittent vital signs monitoring in hospitals: A systematic review and narrative synthesis. Int J Nurs Stud. 2018;84:19-27. doi:10.1016/J.IJNURSTU.2018.04.013.

9. Appelboom G, Camacho E, Abraham ME, Bruce SS, Dumont EL, Zacharia BE, et al. Smart wearable body sensors for patient self-assessment and monitoring. Arch Public Heal. 2014;72:28. doi:10.1186/2049-3258-72-28.

10. Weenk M, van Goor H, Frietman B, Engelen LJ, van Laarhoven CJ, Smit J, et al. Continuous Monitoring of Vital Signs Using Wearable Devices on the General Ward: Pilot Study. JMIR mHealth uHealth. 2017;5:e91. doi:10.2196/mhealth.7208.

11. Sun L, Joshi M, Khan SN, Ashrafian H, Darzi A. Clinical impact of multi-parameter continuous non-invasive monitoring in hospital wards: a systematic review and meta-analysis. J R Soc Med. 2020;113:217-24. doi:10.1177/0141076820925436.

12. Leenen JPL, Leerentveld C, van Dijk JD, van Westreenen HL, Schoonhoven L, Patijn GA. Current Evidence for Continuous Vital Signs Monitoring by Wearable Wireless Devices in Hospitalized Adults: Systematic Review. J Med Internet Res. 2020;22:e18636. doi:10.2196/18636.

13. Naziyok TP, Zeleke AA, Röhrig R. Contactless Patient Monitoring for General Wards: A Systematic Technology Review. Stud Health Technol Inform. 2016;228:707-11.

14. Areia C, Vollam S, Young L, Pimentel M, Thursley N, Gerry S, et al. Assessing ambulatory vital sign monitoring to detect deterioration in hospitalised patients: a systematic review. PROSPERO: CRD42020188633. 2020. https://www.crd.york.ac.uk/prospero/display_record.php?ID=CRD42020188633. Accessed 2 Jan 2021.

15. Page MJ, McKenzie JE, Bossuyt PM, Boutron I, Hoffmann TC, Mulrow CD, et al. The PRISMA 2020 statement: an updated guideline for reporting systematic reviews. BMJ. 2021;372:n71. doi:10.1136/bmj.n71

16. Areia C, Vollam S, Young L, Biggs C, Pimentel M, Santos M, et al. Protocol for a systematic review assessing ambulatory vital sign monitoring impact on deterioration detection and related clinical outcomes in hospitalised patients. BMJ Open. 2021;11. doi:10.1136/bmjopen-2020-047715.

17. Dindo D, Demartines N, Clavien P-A. Classification of surgical complications: a new proposal with evaluation in a cohort of 6336 patients and results of a survey. Ann Surg. 2004;240:205-13.

18. Landis JR, Koch GG. The measurement of observer agreement for categorical data. Biometrics. 1977;33:159-74.

19. Altman D. Practical Statistics for Medical Research. London: Chapman and Hall; 1991.

20. Sterne JAC, Savović J, Page MJ, Elbers RG, Blencowe NS, Boutron I, et al. RoB 2: a revised tool for assessing risk of bias in randomised trials. BMJ. 2019;366:14898. doi:10.1136/bmj.14898.

21. Wells G, Shea B, O'Connell D, Peterson J, Welsh V, Losos M, et al. The Newcastle-Ottawa Scale (NOS) for assessing the quality of nonrandomised studies in meta-analyses. The Ottawa Hospital Research Institute.

22. Sterne JAC, Hernán MA, Reeves BC, Savović J, Berkman ND, Viswanathan M, et al. ROBINS-I: a tool for assessing risk of bias in non-randomised studies of interventions. BMJ. 2016;355:i4919. doi:10.1136/bmj.i4919.

23. Hong QN, Pluye P, Fàbregues S, Bartlett G, Boardman F, Cargo M, et al. Mixed Methods Appraisal Tool (MMAT) Version 2018. McGIII Department of Family Medicine. 2018. http://mixedmethodsappraisaltoolpublic.pbworks.com/w/file/fetch/127425851/MMAT_2018_criteria-manual_2018-04-04.pdf. Accessed 15 Jul 2020

24. The Cochrane Collaboration. Review Manager (RevMan). 2020.

Page 12/18 
25. Deeks J, Higgins J, Altman D. Chapter 10: Analysing data and undertaking meta-analyses. In: Higgins JPT, Thomas J, Chandler J, Cumpston M, Li T, Page MJ, Welch VA. Cochrane Handbook for Systematic Reviews of Interventions version 6.1. Cochrane; 2020.

https://training.cochrane.org/handbook/current/chapter-10.

26. Weller RS, Foard KL, Harwood TN. Evaluation of a wireless, portable, wearable multi-parameter vital signs monitor in hospitalized neurological and neurosurgical patients. J Clin Monit Comput. 2018.

27. Skraastad EJ, Borchgrevink PC, Nilsen TIL, Ræder J. Postoperative quality and safety using Efficacy Safety Score (ESS) and a wireless patient monitoring system at the ward: A randomised controlled study. Acta Anaesthesiol Scand. 2020;64:301-8. doi:https://doi.org/10.1111/aas.13492.

28. PT Higgins J, Li T, K Deeks J. Chapter 6: Choosing effect measures and computing estimates of effect. In: Cochrane Handbook for Systematic Reviews of Interventions version 6.0. 2019. www.training.cochrane.org/handbook.

29. Downey C, Randell R, Brown J, Jayne DG. Continuous versus intermittent vital signs monitoring using a wearable, wireless patch in patients admitted to surgical wards: Pilot cluster randomized controlled trial. J Med Internet Res. 2018;20:e10802. doi:10.2196/10802.

30. Downey CL, Croft J, Ainsworth G, Buckley H, Shinkins B, Randell R, et al. Trial of remote continuous versus intermittent NEWS monitoring after major surgery (TRaCINg): a feasibility randomised controlled trial. Pilot Feasibility Stud. 2020;6:183. doi:10.1186/s40814-020-00709-8.

31. McMaster University. GRADEpro GDT: GRADEpro Guideline Development Tool [Software]. 2020. gradepro.org.

32. Bailey TC, Chen Y, Mao Y, Lu C, Hackmann G, Micek ST, et al. A trial of a real-time alert for clinical deterioration in patients hospitalized on general medical wards. J Hosp Med. 2013;8:236-42.

33. Subbe CP, Duller B, Bellomo R. Effect of an automated notification system for deteriorating ward patients on clinical outcomes. Crit Care. $2017 ; 21: 52$. doi:10.1186/s13054-017-1635-z.

34. Taenzer AH, Pyke JB, McGrath SP, Blike GT. Impact of pulse oximetry surveillance on rescue events and intensive care unit transfers: a before-and-after concurrence study. Anesthesiology. 2010;112:282-7.

35. Duncan HP, Fule B, Rice I, Sitch AJ, Lowe D. Wireless monitoring and real-time adaptive predictive indicator of deterioration. Sci Rep. 2020;10:11366. doi:10.1038/s41598-020-67835-4.

36. Verrillo SC, Cvach M, Hudson KW, Winters BD. Using Continuous Vital Sign Monitoring to Detect Early Deterioration in Adult Postoperative Inpatients. J Nurs Care Qual. 2019;34:107-13. doi:10.1097/NCQ.0000000000000350.

37. Weenk M, Bredie SJ, Koeneman M, Hesselink G, van Goor H, van de Belt TH. Continuous Monitoring of Vital Signs in the General Ward Using Wearable Devices: Randomized Controlled Trial. J Med Internet Res. 2020;22:e15471.

38. Weenk M, Koeneman M, van de Belt TH, Engelen LJLPG, van Goor H, Bredie SJH. Wireless and continuous monitoring of vital signs in patients at the general ward. Resuscitation. 2019;136:47-53.

39. Kisner D, Wilhelm MJ, Messerli MS, Zünd G, Genoni M. Reduced incidence of atrial fibrillation after cardiac surgery by continuous wireless monitoring of oxygen saturation on the normal ward and resultant oxygen therapy for hypoxia. Eur J cardio-thoracic Surg Off J Eur Assoc Cardio-thoracic Surg. 2009;35:111-5.

40. Watkinson PJ, Pimentel MAF, Clifton L, Clifton DA, Vollam S, Young D, et al. Early detection of physiological deterioration in post-surgical patients using wearable technology combined with an integrated monitoring system: a pre- and post-interventional study. medRxiv. 2020;:2020.12.01.20240770. doi:10.1101/2020.12.01.20240770.

41. Monson M, Heuser C, Einerson BD, Esplin I, Snow G, Varner M, et al. Evaluation of an external fetal electrocardiogram monitoring system: a randomized controlled trial. Am J Obstet Gynecol. 2020;223:244.e1-244.e12.

42. Daabiss M. American Society of Anaesthesiologists physical status classification. Indian J Anaesth. 2011;55:111-5. doi:10.4103/0019-5049.79879.

43. Watkinson PJ. Does continuous monitoring of vital signs with an alerting system reduce length of hospital stay in post-operative upper gastro-intestinal surgery patients? ISRCTN. 2017. doi:https://doi.org/10.1186/ISRCTN58660550.

44. Downey CL, Croft J, Buckley H, Randell R, Brown JM, Jayne DG. Trial of Remote Continuous versus Intermittent NEWS monitoring after major surgery (TRaCINg): protocol for a feasibility randomised controlled trial. Pilot Feasibility Stud. 2018;4:112. doi:10.1186/s40814-018-0299-z.

45. Bailey TC. ClinicalTrials.gov NCT01280942: Early Warning System for Clinical Deterioration on General Hospital Wards. U.S. National Library of Medicine. 2011. https://clinicaltrials.gov/ct2/show/NCT01280942.

46. Subbe CP. ClinicalTrials.gov NCT01692847: Examination of the Impact of Better Surveillance and Communication of Patient Deterioration on Patient Related Outcomes. U.S. National Library of Medicine. 2012. https://clinicaltrials.gov/ct2/show/NCT01692847.

47. Reed M. ClinicalTrials.gov NCT03179267: Detection of Deterioration by SNAP40 Versus Standard Monitoring in the ED (SNAP40ED). U.S. National Library of Medicine. 2017. https://clinicaltrials.gov/ct2/show/NCT03179267. Accessed 28 Feb 2021.

48. Mackay J. ClinicalTrials.gov NCT02427828: VRES Study in Two Phases to Monitor Cardiac Surgical Patients Following Discharge From ICU (VRES). U.S. National Library of Medicine. 2015. https://clinicaltrials.gov/ct2/show/record/NCT02427828. Accessed 28 Feb 2021.

49. Joshi M, Archer S, Morbi A, Arora S, Kwasnicki R, Ashrafian H, et al. Short-Term Wearable Sensors for In-Hospital Medical and Surgical Patients: Mixed Methods Analysis of Patient Perspectives. JMIR Perioper Med. 2021;4:e18836. doi:10.2196/18836.

50. Olsson A, Persson A-C, Bartfai A, Boman I-L. Sensor technology more than a support. Scand J Occup Ther. 2018;25:79-87. doi:10.1080/11038128.2017.1293155.

51. Downey CL, Brown JM, Jayne DG, Randell R. Patient attitudes towards remote continuous vital signs monitoring on general surgery wards: An interview study. Int J Med Inform. 2018;114:52-6. doi:10.1016/j.ijmedinf.2018.03.014.

Page $13 / 18$ 
52. de Man FR, Greuters S, Boer C, Veerman DP, Loer SA. Intra-operative monitoring - many alarms with minor impact. Anaesthesia. 2013;68:804-10. doi:https://doi.org/10.1111/anae.12289.

53. Bonafide CP, Lin R, Zander M, Graham CS, Paine CW, Rock W, et al. Association between exposure to nonactionable physiologic monitor alarms and response time in a children's hospital. J Hosp Med. 2015;10:345-51.

54. Drew BJ, Harris P, Zègre-Hemsey JK, Mammone T, Schindler D, Salas-Boni R, et al. Insights into the problem of alarm fatigue with physiologic monitor devices: a comprehensive observational study of consecutive intensive care unit patients. PLoS One. 2014;9:e110274.

55. Görges M, Markewitz BA, Westenskow DR. Improving alarm performance in the medical intensive care unit using delays and clinical context. Anesth Analg. 2009;108:1546-52.

56. Higgins J, Eldridge SM, Li T. Chapter 23: Including variants on randomized trials. In: Higgins JPT, Thomas J, Chandler J, Cumpston M, Li T, Page MJ, Welch VA. Cochrane Handbook for Systematic Reviews of Interventions version 6.1. 2020. https://training.cochrane.org/handbook/current/chapter-23.

57. Posthuma LM, Downey C, Visscher MJ, Ghazali DA, Joshi M, Ashrafian H, et al. Remote wireless vital signs monitoring on the ward for early detection of deteriorating patients: A case series. Int J Nurs Stud. 2020;104:103515. doi:https://doi.org/10.1016/j.ijnurstu.2019.103515.

58. Areia C, Young L, Vollam S, Ede J, Santos M, Tarassenko L, et al. Wearability Testing of Ambulatory Vital Sign Monitoring Devices: Prospective Observational Cohort Study. JMIR Mhealth Uhealth. 2020;8:e20214. doi:10.2196/20214.

59. Areia C, Vollam S, Piper P, King E, Ede J, Young L, et al. Protocol for a prospective, controlled, cross-sectional, diagnostic accuracy study to evaluate the specificity and sensitivity of ambulatory monitoring systems in the prompt detection of hypoxia and during movement. BMJ Open. 2020;10:e034404. doi:10.1136/bmjopen-2019-034404.

60. Monson M. ClinicalTrials.gov NCT03156608: Novii External Fetal Monitoring Device (Novii). U.S. National Library of Medicine. 2017. https://clinicaltrials.gov/ct2/show/NCT03156608. Accessed 27 Feb 2021.

61. Weenk M. ClinicalTrials.gov NCT02933307: Continuous Monitoring on the General Ward. U.S. National Library of Medicine. 2016. https://clinicaltrials.gov/ct2/show/NCT02933307. Accessed 27 Feb 2021.

62. Downey C. Remote vital signs monitoring in surgical patients. ISRCTN. 2016. doi:https://doi.org/10.1186/ISRCTN60999823.

63. Downey C. Making surgery safer: Testing a wireless monitoring patch on general surgery wards. ISRCTN. 2017. doi:https://doi.org/10.1186/ISRCTN16601772.

64. Skraastad EJ. ClinicalTrials.gov NCT03438578: Efficacy Safety Score in Postoperative Management. U.S. National Library of Medicine. 2018. https://clinicaltrials.gov/ct2/show/study/NCT03438578. Accessed 27 Feb 2021.

\section{Figures}

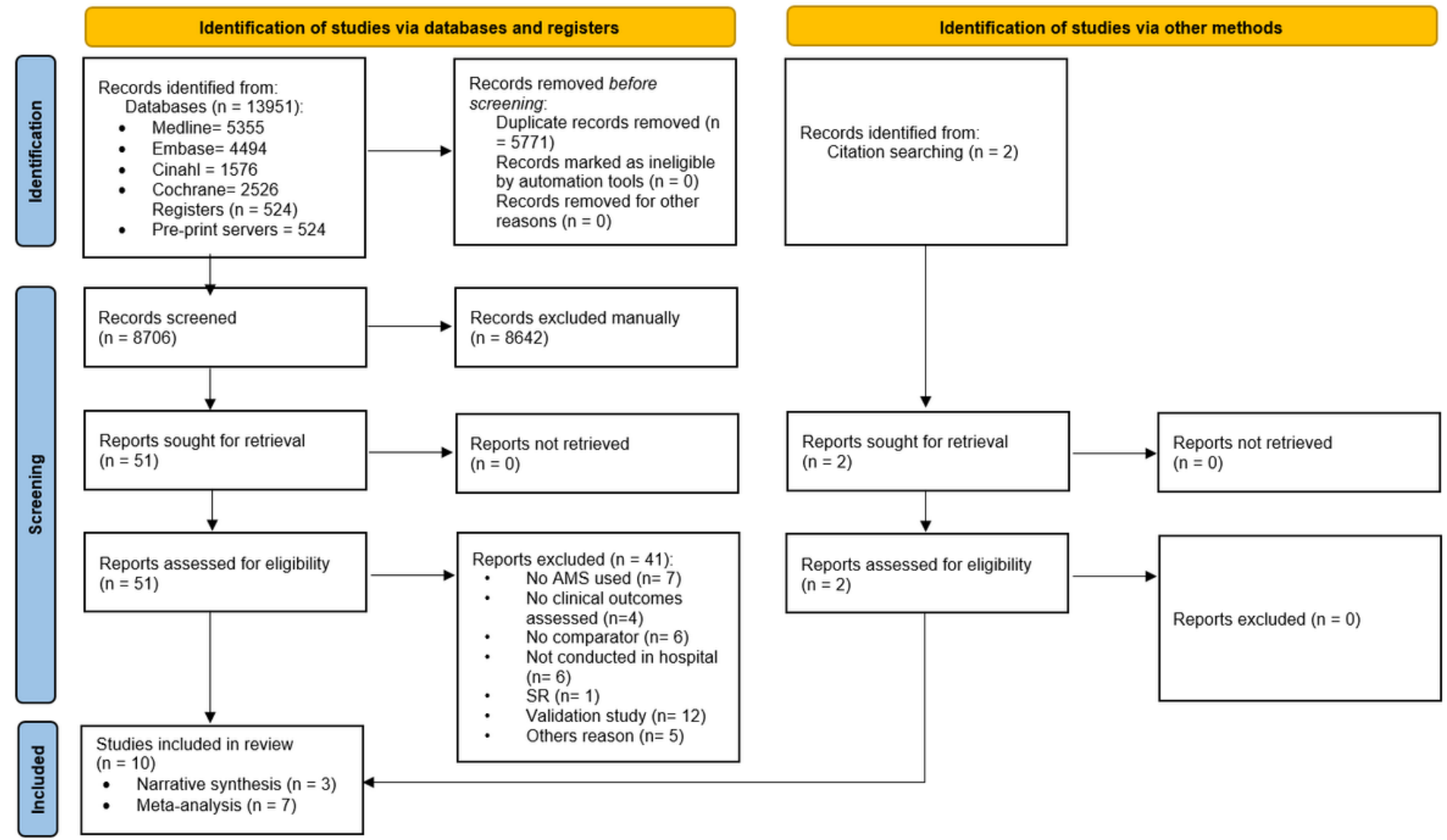

Figure 1 


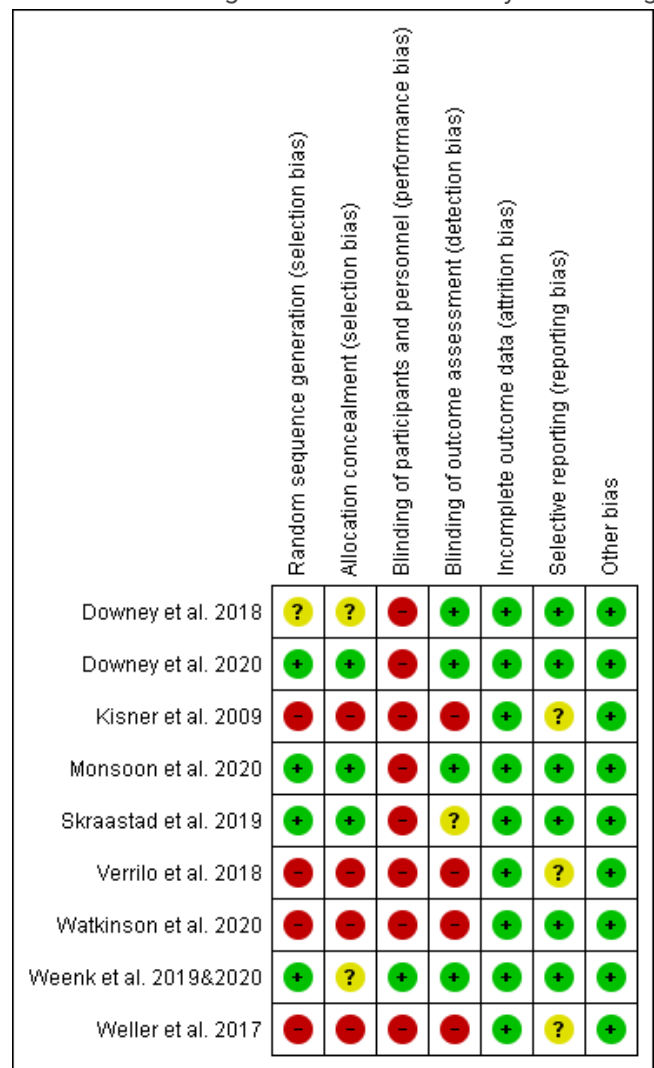

Figure 2

Risk of bias summary: review authors' judgements about each risk of bias item for each included study.

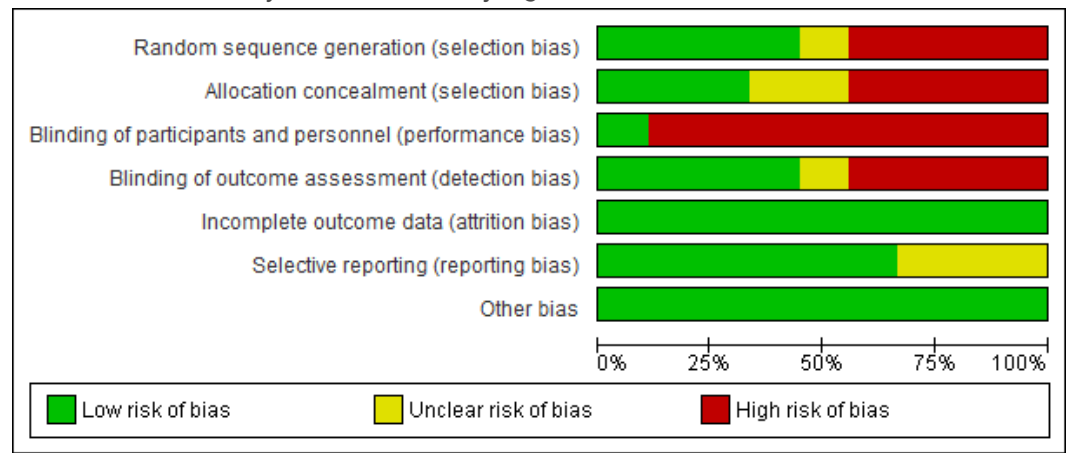

Figure 3

Risk of bias graph: review authors' judgements about each risk of bias item presented as percentages across all included studies. 


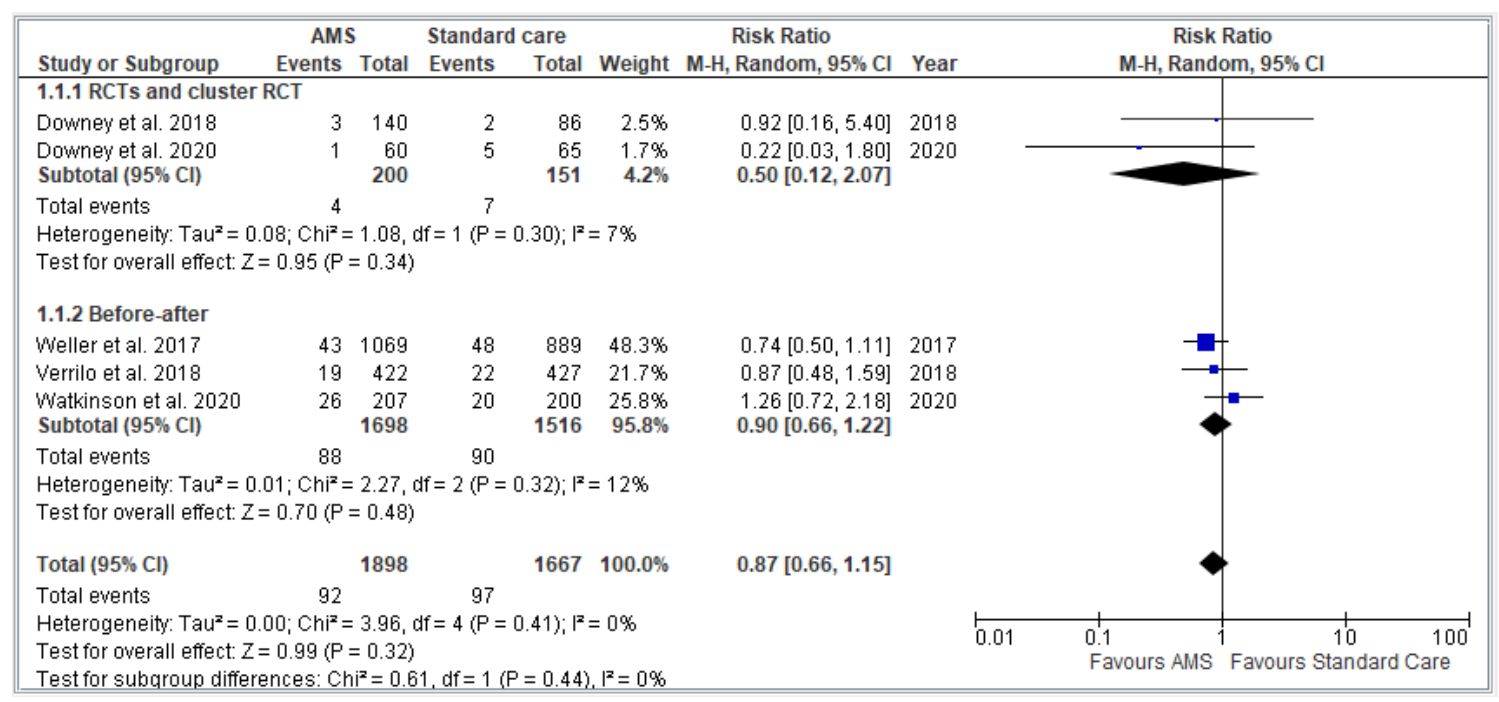

\section{Figure 4}

Meta-analysis forest plots comparing AMS and standard care ICU transfer risk ratio. AMS: ambulatory monitoring systems, Cl: confidence intervals, M-H: Mantel-Haenszel, RCT: randomised controlled trial.

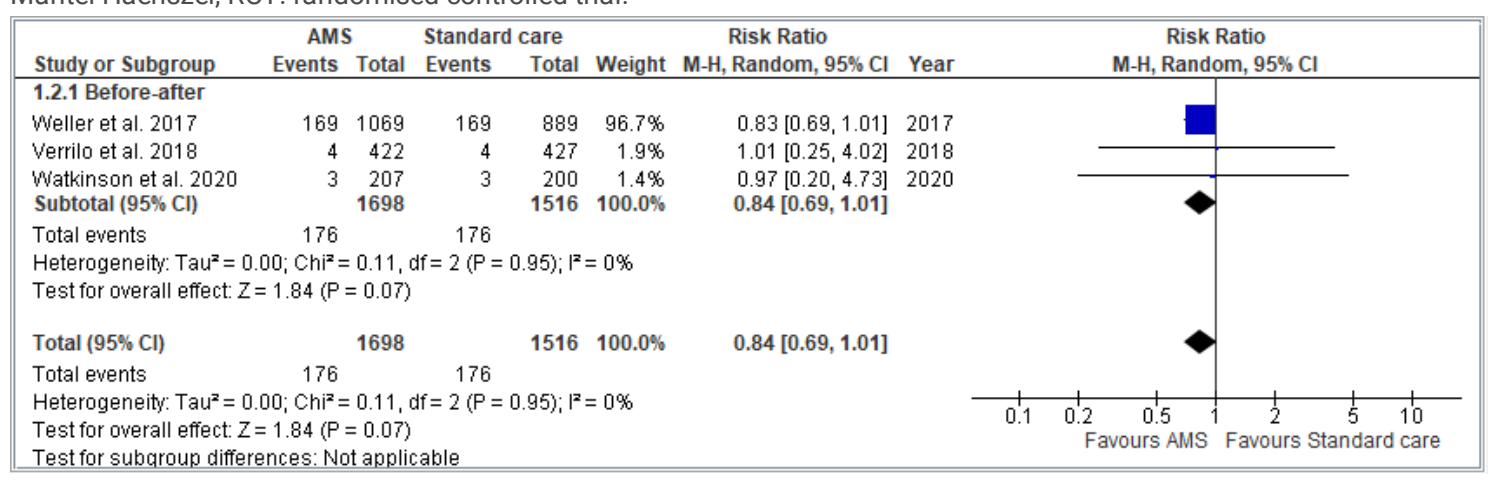

\section{Figure 5}

Meta-analysis forest plots comparing AMS and standard care rapid response or cardiac arrest team activation risk ratio. AMS: ambulatory monitoring systems, Cl: confidence intervals, M-H: Mantel-Haenszel.

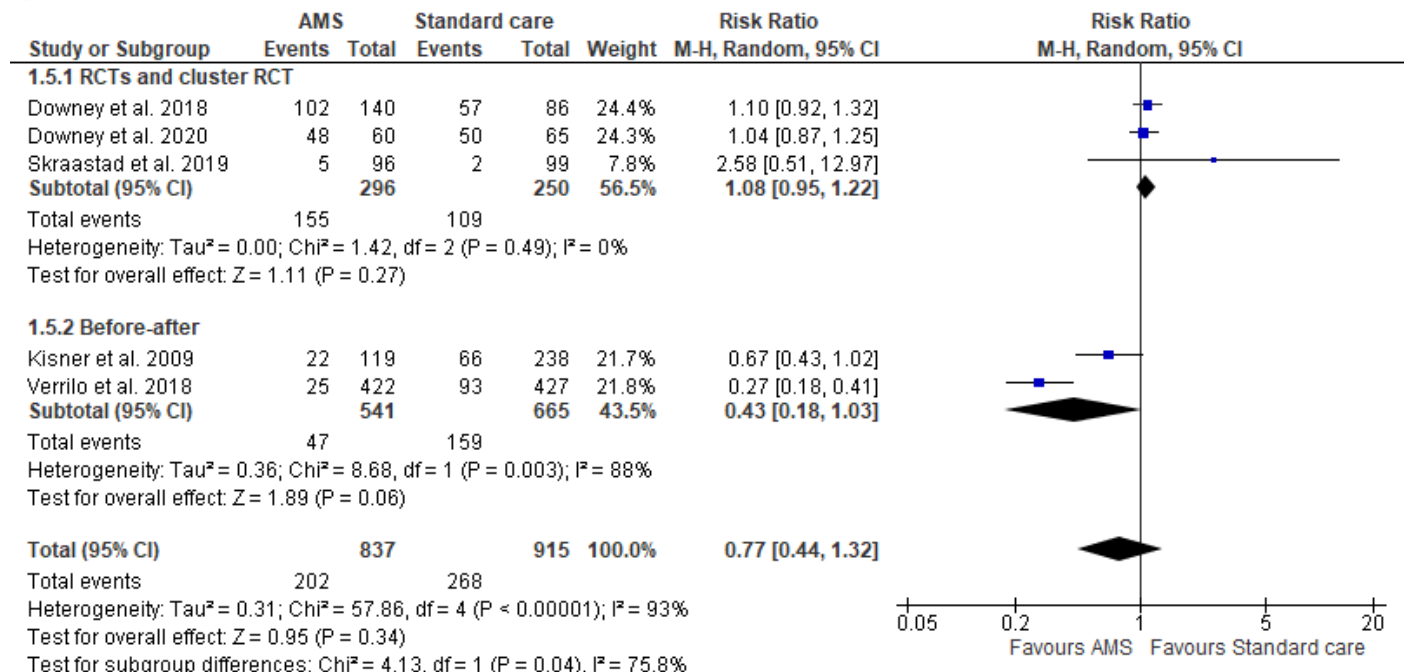

\section{Figure 6}

Meta-analysis forest plots comparing AMS and standard care all complications risk ratio. AMS: ambulatory monitoring systems, $\mathrm{Cl}$ : confidence intervals, $\mathrm{M}-\mathrm{H}$ : Mantel-Haenszel, RCT: randomised controlled trial 


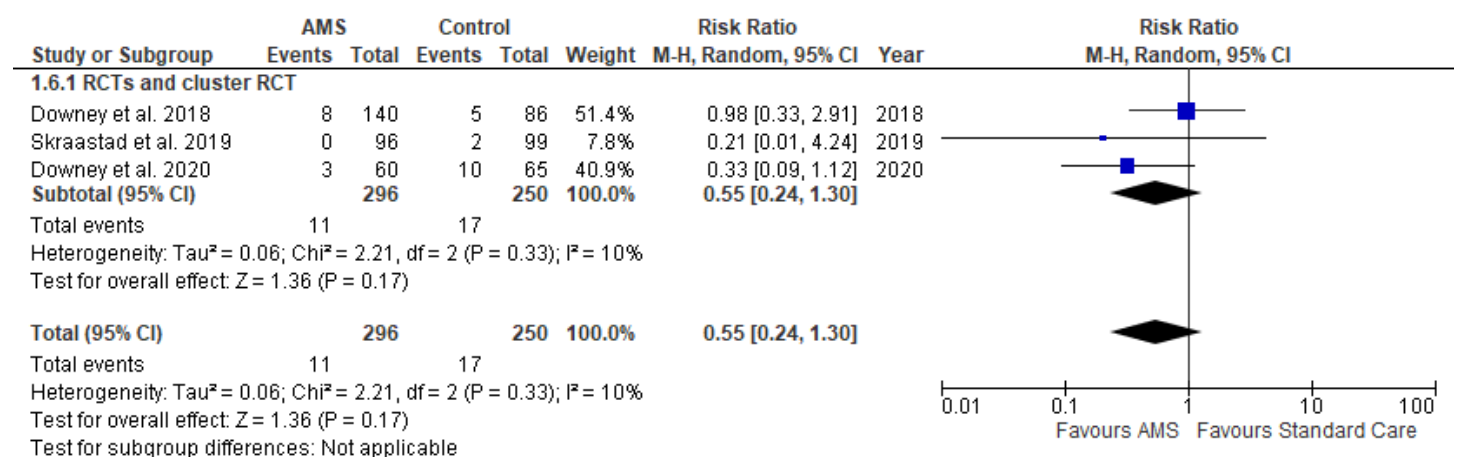

\section{Figure 7}

Meta-analysis forest plots comparing AMS and standard care major complications risk ratio. AMS: ambulatory monitoring systems, Cl: confidence intervals, $\mathrm{M}-\mathrm{H}$ : Mantel-Haenszel, RCT: randomised controlled trial

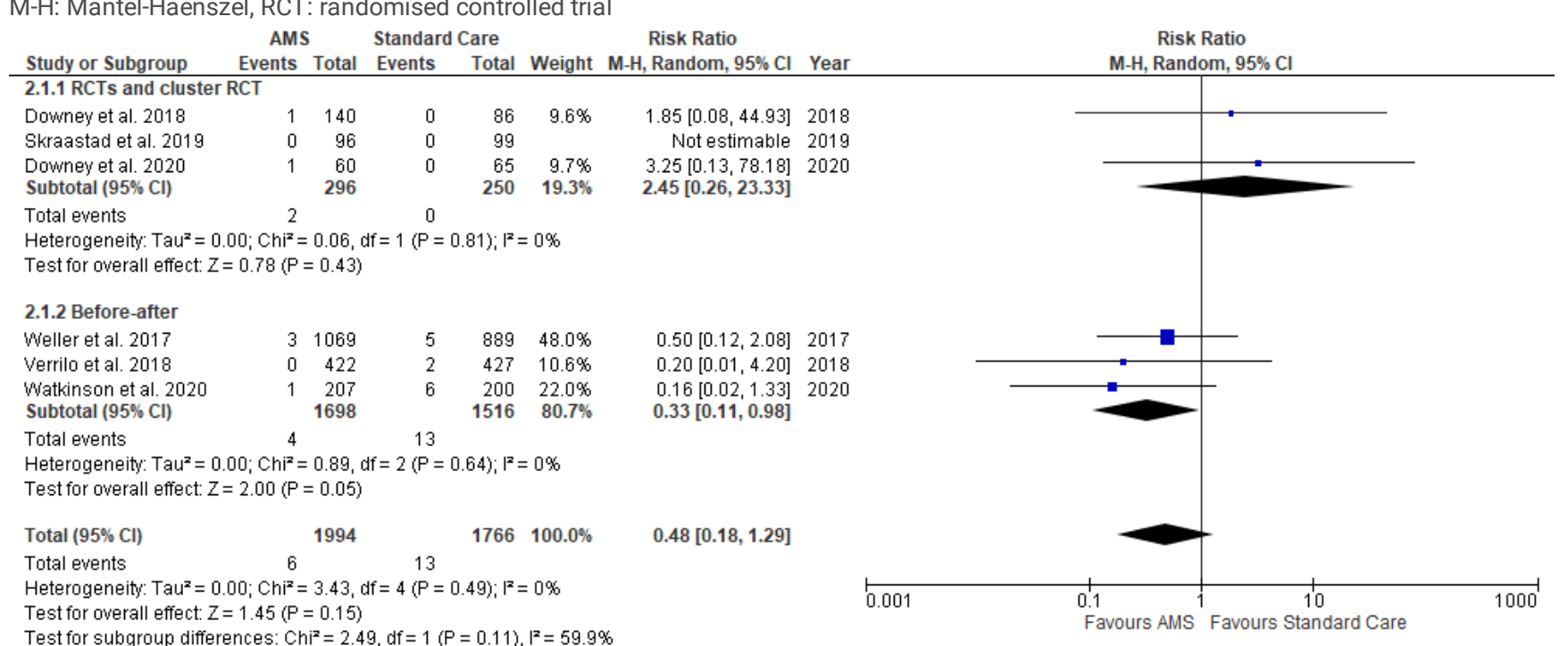

\section{Figure 8}

Meta-analysis forest plots comparing AMS and standard care in-hospital mortality risk ratio. AMS: ambulatory monitoring systems, Cl: confidence intervals, M$\mathrm{H}$ : Mantel-Haenszel, RCT: randomised controlled trial.

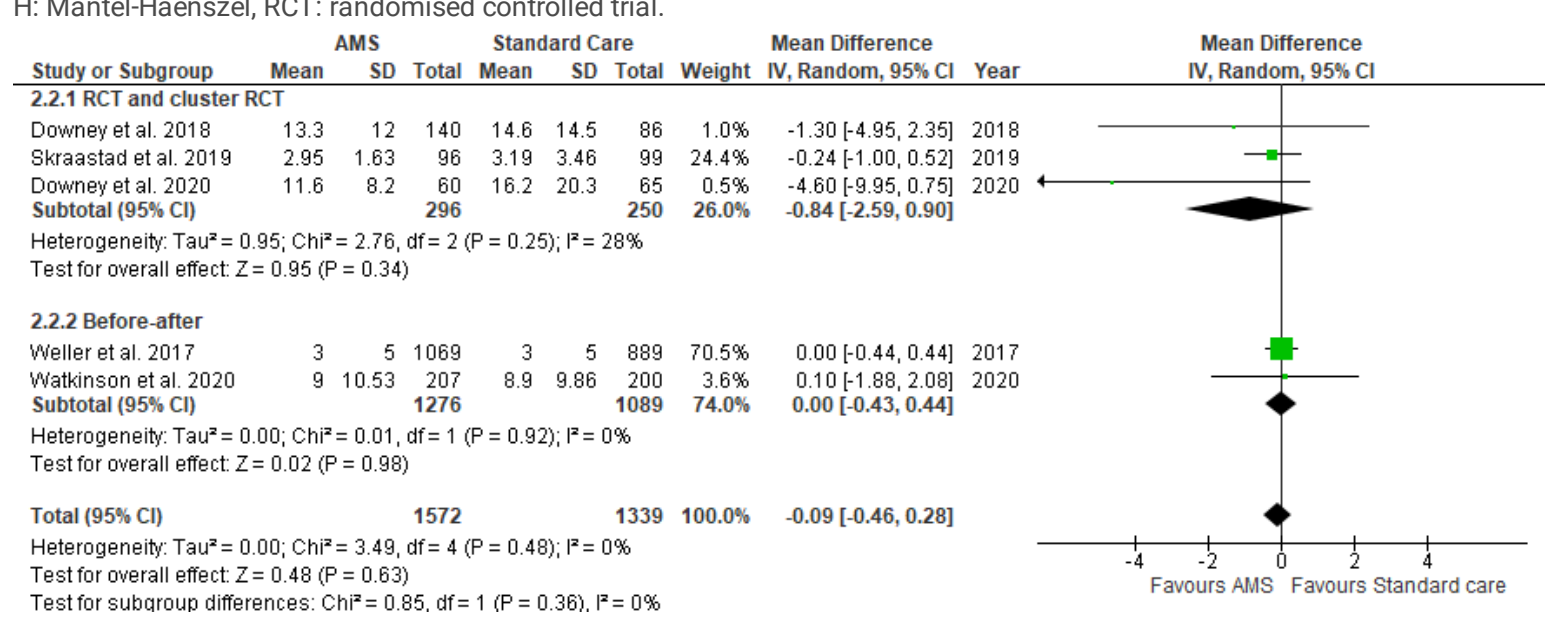

\section{Figure 9}

Meta-analysis forest plots comparing AMS and standard care hospital length of stay mean difference. AMS: ambulatory monitoring systems, Cl: confidence intervals, IV: inverse variance, RCT: randomised controlled trial

\section{Supplementary Files}


This is a list of supplementary files associated with this preprint. Click to download.

- Appendix1.docx

- Appendix2.docx

- Appendix3.docx

- Appendix4.docx

- Appendix5.docx

- Appendix6.docx

- Appendix7.docx

- Appendix8.docx

- GraphicalAbstract.png 